\title{
Functionality in Tool Use in Western Lowland Gorillas (Gorilla gorilla gorilla)
}

\author{
Matthew K. LeFauve ${ }^{1}$ and Susan W. Margulis ${ }^{1 *}$ \\ ${ }^{1}$ Canisius College \\ *Corresponding author (Email: aperesearchers@gmail.com)
}

Citation - LeFauve, M. K., \& Margulis, S. W. (2015). Functionality in tool use in Western lowland gorillas (Gorilla gorilla gorilla). Animal Behavior and Cognition, 2(1), 96-104. doi: 10.12966/abc.02.08.2015

\begin{abstract}
Nonhuman primates are known to use objects as tools. Amongst the great apes, gorillas seem to be the least proficient tool users. Previous research has shown that the western lowland gorillas (Gorilla gorilla gorilla) at the Buffalo Zoo use buckets, given for enrichment, to collect water (Margulis, Steele, \& Kleinfelder, 2012). To further explore the cognitive ability of these gorillas, a study was designed that tested whether the gorillas could distinguish between a functional and a non-functional manipulable object. The gorillas were given four buckets, two of which had holes drilled in the bottom (the "non-functional" bucket). Seventy-eight hours of videotaped data were collected to test the hypothesis that the gorillas could distinguish between the functional and the non-functional buckets for transport of liquids. Overall, gorillas interacted with functional buckets significantly more than with nonfunctional buckets. This pattern was driven largely by the behavior of the oldest adult female. The findings suggest that gorillas have the ability to recognize tool functionality.
\end{abstract}

Keywords - Gorilla, Tool-use, Tool functionality, Object manipulation

Once thought to be a defining feature of Hominins, tool use has now been documented widely across a diverse array of taxa. These include, for example, invertebrates (Mather, 1994), birds (Bird \& Emery, 2009) and non-primate mammals (Bauer, 2001; Chevalier-Skolnikoff \& Liska, 1993; Hall \& Schaller, 1964; Mann \& Patterson, 2013; Thompson et al., 2007). Tool use has been most broadly demonstrated among the non-human primates (see for example Shumaker, Walkup, \& Beck, 2011 for a review). The first studies conducted by Goodall (1964) on chimpanzees (Pan troglodytes) at Gombe Reserve clearly showed chimpanzees modifying branches to extract and consume termites, confirming that tool use was not unique to humans.

Defining tool use has proven to be problematic. Finn, Tregenza, and Norman (2009) considered a case of an octopus carrying a shell for future use to be an example of tool use. In contrast, Jones and Kamil (1973) offered a more specific definition of tool use: "the use of physical objects other than the animal's own body or appendages as a means to extend the physical influence realized by the animal". (p. 1076) Van Lawick-Goodall (1970) defined tool use as "the use of an external object as a functional extension of mouth or beak, hand or claw, in the attainment of an immediate goal. (p. 195)" Beck (1980) refined and expanded on this definition:

The external employment of an unattached environmental object to alter more efficiently the form, position, or condition of another object, another organism, or the user itself when the user holds or carries the tool during or just prior to use and is responsible for the proper and effective orientation of the tool. (p. 10). 


\section{St. Amant and Horton (2008) further modified Beck's definition as follows:}

Tool use is the exertion of control over a freely manipulable external object (the tool) with the goal of (1) altering the physical properties of another object, substance, surface or medium (the target, which may be the tool user or another organism) via a dynamic mechanical interaction, or (2) mediating the flow of information between the tool user and the environment or other organisms in the environment. (p. 1203)

This definition encompasses the greatest range of behaviors that may be considered as examples of tool use (and is the definition adhered to for the present study). For example, Breuer, NdoundouHockemba, and Fishlock (2005) reported a case of a wild Western lowland gorilla (Gorilla gorilla gorilla) using a stick while walking through deep water and concluded that she was using it to gauge the depth of water through which she was walking. Because this occurrence did not involve altering an external object, it would not be considered tool use by most other definitions. However, this behavior did mediate the flow of information and in this sense, would be considered an example of tool use according to St. Amant and Horton's definition.

While tool use has been demonstrated in a wide range of taxa, in some cases it appears to be inflexible, resembling an innate fixed action pattern rather than an innovative or learned behavior. For example archer fish (Toxotidae spp.) shoot water drops at terrestrial prey in order to knock the prey into the water; ant lions (Myrmeleontidae spp.) construct pits into which terrestrial invertebrates may fall and then shower the prey with sand pulling it towards the center of the pit; and woodpecker finches (Camarhynchus pallidus) use cactus spines and twigs to dislodge prey from holes in decaying trees (Alcock, 1972). While these examples may be considered tool use by all standard definitions, they do not necessarily demonstrate any cognitive or behavioral flexibility. More cognitively demanding examples of behavior that would appear to be tool use include crows (Corvus spp.) using twigs that they alter to a certain size to extract food (Clair \& Rutz, 2013; Hunt, 1996), capuchins (Cebus spp.) using a rock and anvil to break a nut (Fragaszy, Izar, Visalberghi, Ottoni, \& de Oliveira, 2004; Mannu \& Ottoni, 2009; Moura \& Lee, 2004), and chimpanzees termite fishing (Van Lawick-Goodall, 1973; Seed \& Bryne, 2010) and demonstrating cultural variation in tool-use patterns (Whiten et al., 1999). Other examples of extractive foraging have been demonstrated in orangutans (Pongo pygmaeus, Hirata \& Ohashi, 2003; van Schaik, Deaner, \& Merrill, 1999).

Van Schaik et al. (1999) described six different categories of tool use observed in wild primates. These ranged from the simplest (dislodging objects) to the most complex (extractive foraging). Extractive foraging can be defined as "the exploitation of hidden or embedded foods, generally any food that is not visible to the naked eye"' (Xiang, Liang, Nie, \& Li, 2013, p. 389). Van Schaik et al. (1999) further suggested that gorillas and bonobos were less likely to use tools in the wild due to their foliage-heavy diet. While gorillas are known to engage in complex manipulation in order to safely consume leaves of stinging plants (Byrne, 1996; Byrne \& Byrne, 1993), extractive foraging is not as significant a component of their foraging strategy, as it is for chimpanzees and orangutans.

Call (2013) suggested that flexible tool-use is associated with novel solutions, and "is understood as the ability to adapt to new situations with innovative solutions." (p. 17) Shumaker et al. (2011) made a distinction between tool-use and intelligent tool-use, indicating that a higher level of cognitive awareness does not appear to be essential for certain types of tool-use. However, the ability to determine the functionality of a potential tool is believed to require higher mental functioning. Objects may have some physical properties that are static or unchanging, while other properties may vary. If a property of an object is variable, it changes when the task it achieves changes (Santos, Miller, \& Hauser, 2003). Cottontopped tamarins (Saguinus oedipus) and rhesus macaques (Macaca mulatta) reacted differently to variable tool properties, indicating that they were able to follow a changing set of parameters to make a decision about an object and its suitability for use in a specific task. Santos et al. (2003) used a rake-pull apparatus. In this method, known as expectancy violation, a non-human animal is given access to a set of two rakes that are positioned in such a way as to pull a reward towards the subject, pull the reward into a 
hole, or miss the reward completely. While both species attended to tool shape as an object property, $S$. oedipus but not M. mulatta used tool orientation as a functional characteristic (Santos et al., 2003). Herrmann, Wobber, and Call (2008) stated that while primates may understand that the object has changed and that it has variable properties, not all primates will realize that the outcome of the use of the changed object will also be different. For example, in a study done on chimpanzees, McGrew (1974) provided evidence that it is necessary for a chimpanzee to have a mental representation of what a tool should look like for a specific task and how to modify inadequate ones. Based on the stringent tool requirements for ant-dipping, McGrew concluded that chimpanzees used a cognitive model to represent the appropriate length, diameter and shape for successful ant-dipping. This mental representation of what a functional tool looks like supports the notion that the chimpanzee may be able to understand the changing properties of an object that does not stay the same throughout time and allow different characteristics of items to dictate what the item can be used for, if it can be used at all.

Understanding tool functionality should lead to a preference for functional tools and has been demonstrated in orangutans (Pongo pygmaeus) and gorillas (Gorilla gorilla) (Mulcahy, Call, \& Dunbar, 2005). Subjects were required to select a tool of appropriate length to retrieve an out-of-reach object, requiring that they mentally represent both the length of the tool and the distance to the reward object. Cunningham, Anderson, and Mootnick (2006) used a rake pull task similar to Santos, and demonstrated that hoolock gibbons (Hoolock hoolock) appeared to understand the physical relationship between an object and its environment, while concurrently exhibiting the capacity for tool use.

Research in zoological settings has begun to explore tool use in captive populations of great apes, including gorillas. Several early studies (Fontaine, Moisson, \& Wickings, 1995; Natale, Poti', \& Spinozzi, 1988; Pouydebat, Berge, Gorce, \& Coppens, 2005) included anecdotal examples of spontaneous tool use in captive gorillas, and in some cases provided limited empirical support. A survey conducted by Parker, Mitchell, \& Miles (1999) found that 93\% of zoo-housed gorillas performed at least one type of tool use consistent with the definition proposed by Shumaker et al., (2011). A more recent study by Lonsdorf, Ross, Linick, Milstein, and Melber (2009) at the Lincoln Park Zoo found that gorillas were able to engage in an extractive foraging task, but did so less often and less successfully than chimpanzees. Haslam (2013) provided a more recent synthesis of tool use in zoo-housed primates, including gorillas, and suggested that the captive environment may support the occurrence of tool-use in species that may not regularly exhibit it in the wild.

Previous research documented an example of spontaneous tool use among the Western lowland gorillas at the Buffalo Zoological Gardens (Margulis et al., 2012). The gorillas used a bucket that had been provided as enrichment as a tool to collect and drink water. The behavior was observed on numerous occasions and was performed by more than one animal.

The present study investigated whether the gorillas could determine the functionality of the buckets they were using by providing both functional and nonfunctional buckets. Given their phylogenetic relationship to proficient tool-using and tool-making species, it was hypothesized that the gorillas would be able to distinguish a functional tool from a non-functional tool.

\section{Method}

\section{Subjects and Housing}

The individual gorillas in this study were all captive born and housed at the Buffalo Zoological Gardens. The troop consisted of one adult male, (Koga), two adult females (Sidney and Lily) and one juvenile female (Amari). Only the three adults were included in the study. In addition to gorillas, the exhibit housed three species of African birds: Lady Ross' Plantain Eater (Musophaga rossae), Speckled Mousebird (Colius striatus), and the Superb Starling (Lamprotornis superbus).

The exhibit was indoors and measured approximately $20 \mathrm{~m}$ by $20 \mathrm{~m}$. In the eastern corner of the exhibit there was a waterfall that emptied into a standing pool. The exhibit also contained a metal climbing structure, with a net and a hammock composed from woven fire hoses. There were several areas 
in which the gorillas could hide if they wished to be out of view of the public.

\section{Procedures}

All observations were recorded using two Sanyo high-resolution cameras (modelVCC-6584DN) connected to two Mace Security Systems model 400-RT2 DVRs. Two days per week during the week, for eight weeks, from July 20, 2011 to September 9, 2011, the gorillas were given four empty five-gallon plastic buckets. Buckets were placed in the exhibit prior to the animals entering the exhibit each morning (shortly before 10AM). The first two weeks comprised a baseline period, during which all buckets were intact, or functional. This procedure replicated the previously experiment by Margulis et al. (2012). This baseline period was conducted to reintroduce the gorillas to the buckets (which were used intermittently as enrichment), as well as confirming that the gorillas still interacted with the buckets and used them to transport water and/or other materials. Once the baseline period (all functional buckets) was concluded, gorillas were always given two functional and two non-functional buckets. The non-functional buckets had holes drilled in the bottom. Thus, they would not hold water. In order to facilitate identification of the bucket types on video, intact buckets were marked with a red " $\mathrm{X}$ " and non-functional buckets were marked with a red "O" for the first half of the study. Midway through, we switched the markings, such that functional buckets were marked with "O" and non-functional with "X". This was done to avoid any potential bias due to marking.

The DVR was set to record from 9:30:00 AM to 4:00:00 PM each recording day. Our dataset consisted of $14 \mathrm{hrs}$ of baseline data and 78 hours of experimental data. The videos were scored by trained observers who had reached an acceptable level of reliability $(\geq 90 \%$ agreement based on independent scoring of $30 \mathrm{~min}$ of videotape). Each 7.5-hr day of recording was considered to be a trial. All video was observed in real-time, and all-occurrence sampling was used to tabulate all instances of bucket use. These data were entered directly into an excel spreadsheet. Because all trials were of equal duration ( $7.5 \mathrm{hrs})$, and total number of occurrences was tabulated, all $78 \mathrm{hrs}$ of experimental data were combined for analysis. The ethogram for the study is presented in Table 1.

Table 1

Ethogram of Behaviors Analyzed in the Present Study

\begin{tabular}{lll}
\hline Behavior & Description & Observed Occurrences \\
\hline C (Carry Bucket) & $\begin{array}{l}\text { Picking up the bucket and moving it to } \\
\text { another position in the exhibit }\end{array}$ & 63 Observations \\
WA (Water Activities) & $\begin{array}{l}\text { Putting water into the bucket by holding it } \\
\text { under the waterfall or submerging }\end{array}$ & 11 Observations \\
PB (Pick Up/Hold Bucket) & $\begin{array}{l}\text { Pick up and/or hold bucket without moving } \\
\text { it }\end{array}$ & 50 Observations \\
DR (Drink) & $\begin{array}{l}\text { Lifting bucket with water up to mouth } \\
\text { RR (Regurgitate and Reingest) }\end{array}$ & 21 Observations \\
& $\begin{array}{l}\text { Regurgitate and Reingest on top of or into } \\
\text { bucket }\end{array}$ & 74 Observations \\
\hline
\end{tabular}

Behaviors that occurred too rarely for individual analysis (Carry in Hand, Carry in Mouth, Put Face in Bucket, Throw Bucket, Put Food in Bucket) were not included. Behaviors for which bucket functionality was not a factor (Contact Bucket, Invert Bucket, Sit/Lean on Bucket, Reach into Bucket) were also omitted. A total of 219 behavioral occurrences were included for analysis. 


\section{Data Analysis}

A total of 450 interactions with the buckets were observed. Of those, 219 interactions with buckets were analyzed. Only behaviors that involved picking up, carrying, or transporting materials in the bucket were included. Simple contact with bucket was omitted. The total number of occurrences of each behavior over each day of videotaping was recorded. A Chi square test was used to determine if there were differences amongst individuals during the experimental period with respect to which bucket type they used. For all behaviors that occurred frequently enough to run statistical analyses (at least 10 times), the null hypothesis was that the gorillas would not be able to distinguish a functional tool from a nonfunctional tool. Alpha was set at 0.05 .

\section{Results}

When data on all three gorillas were combined, there were no significant differences in choice of functional versus non-functional buckets for the following behaviors: "Pick Up/Hold" $\left(\chi^{2}=2.00, d f=1, p\right.$ $=0.157$; Figure 1A), "Drink" $\left(\chi^{2}=0.0476, d f=1, p=0.827\right.$; Figure 1B $)$, and "Water Activities" $\left(\chi^{2}=1\right.$, $d f=1.00, p=0.132 ;$ Figure 1C).

There were significant differences for the following behaviors: "Carry Bucket" $\left(\chi^{2}=6.667, d f=\right.$ 1, $p=0.0322$; Figure 1D), "Regurgitate and Re-ingest" ( $\chi^{2}=39.405, d f=1, p=0.001$; Figure 1E), and Total Bucket Behaviors $\left(\chi^{2}=19.6366, d f=1, p=<0.001\right.$; Figure 1F). In all cases, the functional buckets were used significantly more than the non-functional buckets.

There were also significant individual differences. Koga's "Regurgitate and Re-ingest" use of the functional bucket was significant $\left(\chi^{2}=5.024, d f=1, p=0.025\right)$ and his total bucket behavior was significant $\left(\chi^{2}=4.00, d f=1, p=0.046\right)$. Sidney's "Regurgitate and Re-ingest" usage of the functional bucket was significant $\left(\chi^{2}=39.405, d f=1, p<0.001\right)$ and Sidney's "Carry Bucket" usage of the functional bucket approached significance $\left(\chi^{2}=3.571, d f=1, p=0.059\right)$. Sidney's total bucket behavior was also significant $\left(\chi^{2}=35.705, d f=1, p<0.001\right)$. Interestingly, Lily did not show a significant trend towards use of either functional or nonfunctional buckets.

\section{Discussion}

The results suggest considerable individual variability in tool use patterns and detection of tool functionality. Nevertheless, the findings support the notion that gorillas have the capacity to discriminate a functional from a non-functional tool. Earlier research (Margulis et al., 2012) demonstrated gorillas in a captive environment have the capacity to use buckets given as enrichment as tools, but there were significant individual differences. These differences were attributed to age, sex, and reproductive status. Specifically, Lily was the most frequent bucket-user in the 2012 study, but this was not the case in the present study. Sidney was observed to use buckets significantly more than Lily, and showed a clear preference for functional buckets. During the 2012 study, Sidney was pregnant, and her overall behavior patterns differed significantly from her non-pregnancy activity patterns (LaDue, Madden, Perkes-Smith, \& Margulis, 2014). It is possible that her altered activity budget may have led to a lower-than-normal level of interaction with enrichment items during the 2012 study. The present findings may therefore provide a better representation of her bucket-usage patterns.

Although Koga showed a significant preference for use of functional buckets, his use of the buckets differed substantially from the types of use that the females engaged in, and most often involved behaviors that we were unable to analyze quantitatively due to their rarity or the nature of the behavior. His most common interaction with the bucket was to use it for agonistic displays, a behavior that, while considered tool use by accepted definitions, does not require functionality discrimination of the tool. Koga was observed being in contact with, hitting, and throwing the bucket in the majority of his observed interactions, but these were not behaviors for which bucket functionality was irrelevant. Throwing objects as an agonistic display has been observed in gorillas in the wild (Wittiger \& Sunderland-Groves, 2007) 
and in captivity (Fontaine et al., 1995).
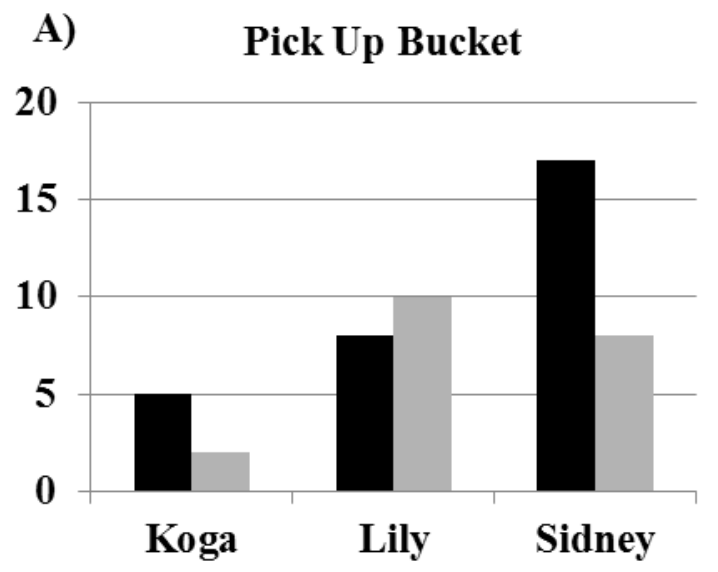

C)

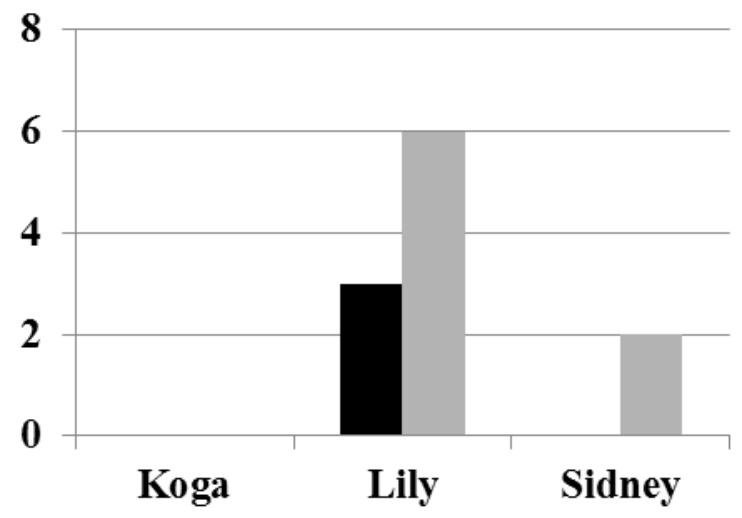

E) Regurgitate and Reingest

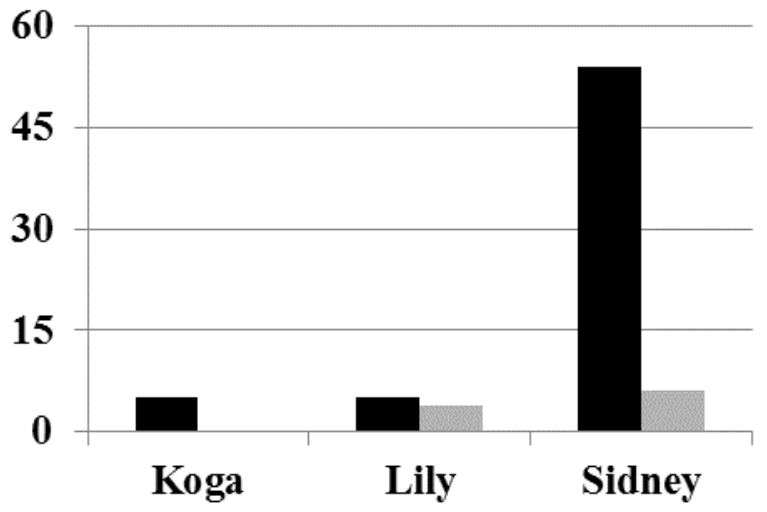

B)

Drink
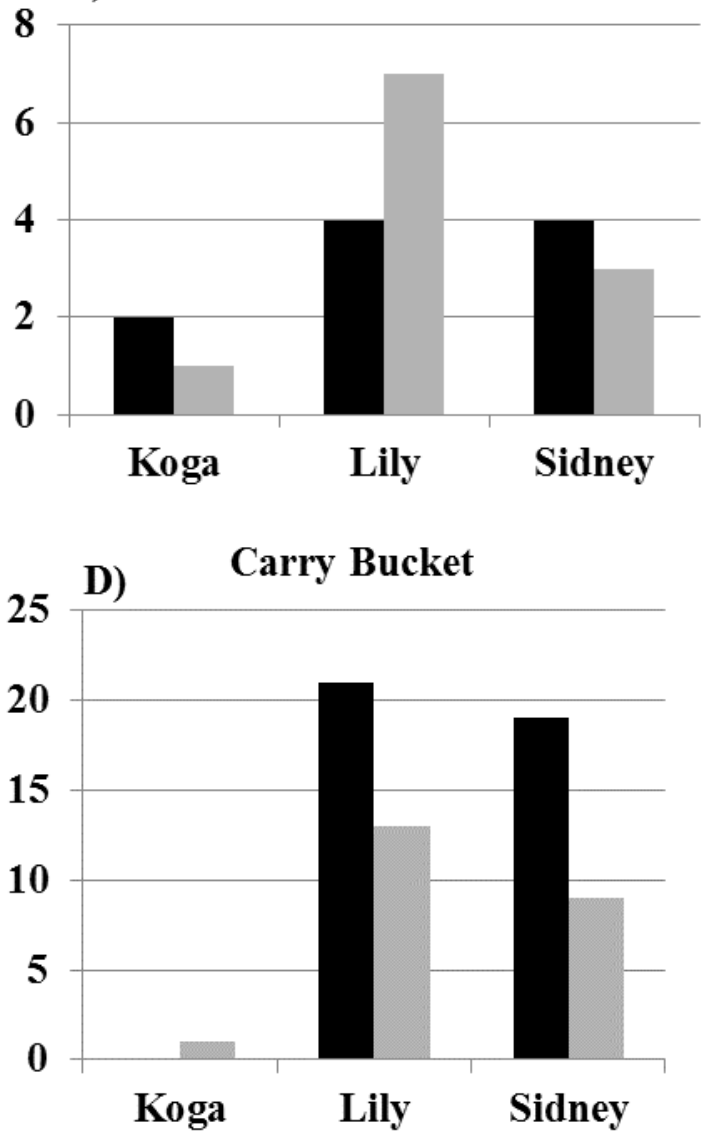

F) Total Bucket Usage

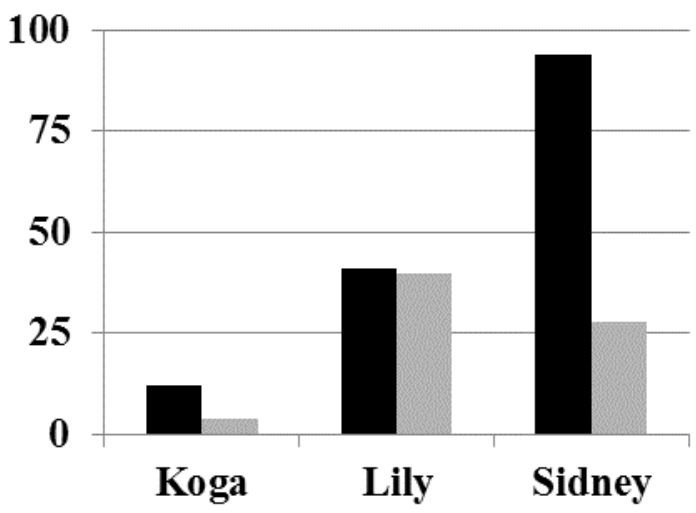

Figure 1. Frequency of bucket usage behaviors by three gorillas (x axis): A. Pick up bucket; B. Drink from bucket; C. Water activities; D. Carry; E. Regurgitate and Reingest; F. Total bucket usage. The Y axis indicates number of occurrence of the behavior. Black bars = functional buckets; gray bars = non-functional buckets. 
Koga was not observed to fill the bucket, but was observed on three occasions drinking from an already filled bucket. On two instances, he was observed to displace one of the females from her bucket after she had filled it (or tried to fill it if it was nonfunctional). It is possible that Koga's significant preference for functional buckets is an artifact of his tendency to displace females from their buckets, and may not represent a true preference for functional buckets. In addition, the ways in which Koga interacted with buckets were not impacted by the presumptive functionality of the bucket. That is, throwing or hitting a non-functional bucket was just as effective a display as throwing a functional bucket.

The gorillas had constant access to a waterfall with a small pool at the bottom, as well as access to a water spigot. Most commonly, the gorillas were observed to drink either by putting their mouth to the standing pool, using their hand to scoop up water, or putting their mouth to the spigot. In those instances in which they used a bucket, animals either held it under the waterfall or submerged the bucket in the pool at the base of the waterfall. In the present study, all individuals had access to the buckets, and yet the water behaviors - filling the bucket, and using it to drink water-were not common behaviors, occurring only 32 times during this investigation. The addition of buckets that did not hold water, and the consequent reduction in reinforcement (successful transport of liquid and/or consumption of liquid) for continuing to use the buckets in this manner, may have led to an extinction of this particular use of the buckets.

When engaging in "water activities," or filling the bucket at the waterfall, Sidney and Lily collected water differently. Lily tended to hold the bucket under the waterfall and Sidney tended to submerge it in the pool at the bottom of the waterfall. Both methods worked, as long as they were using a functional bucket. When filling the bucket, all gorillas proceeded to attempt to drink, however this was only successful if the bucket was functional. Sidney chose functional buckets nearly three times as often as non-functional buckets. Sidney's use of buckets for the "Regurgitate and Re-ingest" behavior provides a strong example of discriminating the non-functional bucket from the functional bucket. Regurgitation and re-ingestion occurs frequently in zoo-housed gorillas and has been the focus of several studies (see Lukas, 1999 for review). Sidney frequently used the buckets to contain the regurgitant. She consistently chose the functional bucket after one occurrence of losing the regurgitant through the holes in the nonfunctional bucket. By collecting and transporting the regurgitant in the bucket, she did not lose any of the regurgitant through runoff or theft by the other gorillas in the troop.

These findings show that gorillas have the cognitive flexibility required for tool use, supports the conclusion that they have the capacity for tool use if there is situational need, and shows that they have the capability to determine tool functionality. One female showed a clear and significant preference for functional tools and one did not. Because the silverback often displaced females from their tools, his pattern of tool use may in fact be more dependent on the female's and thus may not reflect his own ability to discriminate tools based on functionality. While our findings are preliminary, based on a single gorilla troop, they suggest that, like other great apes, gorillas have the ability to identify the properties of tools and use them in appropriate and often innovative ways. More data from other zoo-housed groups of gorillas, as well as other apes, would further support these conclusions.

\section{Acknowledgments}

We are grateful to the staff of the Buffalo Zoological Gardens for their assistance and support throughout this study. In particular, we thank Dr. Donna Fernandes, Jerry Aquilina, and the keepers of the Gorilla Forest exhibit for their assistance. This study was approved by the IACUC of Canisius College and Research Committee of the Buffalo Zoo. ML was supported by a Canisius Earning Excellence Program Fellowship. We thank two anonymous reviewers for helpful comments on an earlier version of this manuscript.

\section{References}

Alcock, J. (1972). The evolution of the use of tools by feeding animals. Evolution. 26, 464-473. 
Bauer, H. (2001). Use of tools by lions in Waza National Park, Cameroon. African Journal of Ecology, $39,317$.

Beck, B. B. (1980). Animal tool behavior. New York: Garland STM Press.

Bird, C. D., \& Emery, N. J. (2009). Insightful problem solving and creative tool modification by captive nontoolusing rooks. Proceedings of the National Academy of Sciences, 106, 10370-10375.

Breuer, T., Ndoundou-Hockemba, M., \& Fishlock, V. (2005). First observation of tool use in wild gorillas. PLoS Biol, 3, e380.

Byrne, R. W. (1996). The misunderstood ape; the cognitive skills of the gorilla. In A. E. Russon, K. A. Bard, \& S. Taylor Parker (Eds.), Reaching into thought: The minds of the great apes (pp. 111-130). Cambridge UK: Cambridge University Press.

Byrne, R., \& Byrne, J. (1993). Complex leaf-gathering skills of mountain gorillas: Variability and standardization. American Journal of Primatology, 31, 241-261.

Call, J. (2013). Three ingredients for becoming a creative tool user. In C. M. Sanz, J. Call, \& C. Boesch (Eds.). Tool Use in Animals: Cognition and ecology (pp. 3-20). Cambridge UK: Cambridge University Press.

Chevalier-Skolnikoff, S., \& Liska, J. (1993). Tool use by wild and captive elephants. Animal Behaviour, 46, 209219.

Cunningham, C. L., Anderson, J. R., \& Mootnick, A. R. (2006). Object manipulation to obtain a food reward in hoolock gibbons, Bunopithecus hoolock. Animal Behaviour, e, 621-629.

Clair, J. J. H. S., \& Rutz, C. (2013). New Caledonian crows attend to multiple functional properties of complex tools. Philosophical Transactions of the Royal Society B: Biological Sciences, 368, 20120415.

Finn, J., Tregenza, T., \& Norman, M. (2009). Defensive tool use in a coconut-carrying octopus. Current Biology, 19, R1069-R1070.

Fontaine, B., Moisson, P., \& Wickings, E. (1995). Observations of spontaneous tool making and tool use in a captive group of Western lowland gorillas (Gorilla gorilla gorilla). Folia Primatologica, 65, $219-223$.

Fragaszy, D., Izar, P., Visalberghi, E., Ottoni, E. B., \& de Oliveira, M. G. (2004). Wild capuchin monkeys (Cebus libidinosus) use anvils and stone pounding tools. American Journal of Primatology, 64, 359-366.

Goodall, J. (1964). Tool-using and aimed throwing in a community of free-living chimpanzees. Nature, 201, 12641266.

Hall, K., \& Schaller, G. (1964). Tool using behavior of the California sea otter. Journal of Mammalology, 45, 287298.

Haslam, M. (2013). "Captivity bias" in animal tool use and its implications for the evolution of hominin technology. Philosophical Transactions of the Royal Society B: Biological Sciences, 368, 20120421.

Herrmann, E., Wobber, V., \& Call, J. (2008). Great apes' (Pan troglodytes, Pan paniscus, Gorilla gorilla, Pongo pygmaeus) understanding of tool functional properties after limited experience. Journal of Comparative Psychology, 122, 220-230.

Hirata, S., \& Ohashi, G. (2003). An experimental study of tool use in orangutans. Reichorui kenkyu, 19, 87-95.

Hunt, G. R. (1996). Manufacture and use of hook-tools by New Caledonian crows. Nature, 379, $249-251$.

Jones, T., \& Kamil, A. (1973). Tool-making and tool-using in the Northern blue jay. Science, 180, $1076-1078$.

LaDue, C., Madden, M., Perkes-Smith, L., \& Margulis, S. (2014). Behavioral changes associated with pregnancy and infant development in captive gorillas. Animal Keepers Forum, 41, 80-83.

Lukas, K. E. (1999). A review of nutritional and motivational factors contributing to the performance of regurgitation and reingestion in captive lowland gorillas (Gorilla gorilla gorilla). Applied Animal Behaviour Science, 63, 237-249.

Lonsdorf, E. V., Ross, S. R., Linick, S. A., Milstein, M. S., \& Melber, T. N. (2009). An experimental, comparative investigation of tool use in chimpanzees and gorillas. Animal Behaviour, 77, 1119-1126.

Mann, J., \& Patterson, E. M. (2013). Tool use by aquatic animals. Philosophical Transactions of the Royal Society B: Biological Sciences, 368, 20120424.

Mannu, M., \& Ottoni, E. B. (2009). The enhanced tool-kit of two groups of wild bearded capuchin monkeys in the Caatinga: Tool making, associative use, and secondary tools. American Journal of Primatology, 71, 242251.

Margulis, S. W., Steele, G. R., \& Kleinfelder, R. E. (2012). Use of buckets as tools by Western lowland gorillas. Zoo Biology, 31, 260-266.

Mather, J. A. (1994). "Home" choice and modification by juvenile Octopus vulgaris (Mollusca: Cephalopoda): specialized intelligence and tool use? Journal of Zoology, 233, 359-368.

McGrew, W. C. (1974). Tool use by wild chimpanzees in feeding upon driver ants. Journal of Human Evolution, 3 , 501-508.

Moura, A., \& Lee, P. (2004). Capuchin stone tool use in Caatinga dry forest. Science, 306, 1909. 
Mulcahy, N. J., Call, J., \& Dunbar, R. I. M. (2005). Gorillas (Gorilla gorilla) and orangutans (Pongo pygmaeus) encode relevant problem features in a tool-using task. Journal of Comparative Psychology, 119, 23-32.

Natale, F., Poti', P., \& Spinozzi, G. (1988). Development of tool use in a macaque and a gorilla. Primates, 29, 413416.

Parker, S. T., Mitchell, R. W., \& Miles, H. L. (1999). The mentalities of gorillas and orangutans: Comparative perspectives. Cambridge UK: Cambridge University Press.

Pouydebat, E., Berge, C., Gorce, P., \& Coppens, Y. (2005). Use and manufacture of tools to extract food by captive Gorilla gorilla gorilla: Experimental approach. Folia Primatologica, 76, 180-183.

Santos, L. R., Miller, C. T., \& Hauser, M. D. (2003). Representing tools: How two non-human primate species distinguish between the functionally relevant and irrelevant features of a tool. Animal Cognition, 6, 269281.

Seed, A., \& Byrne, R. (2010). Animal tool-use. Current Biology, 20, R1032-R1039.

Shumaker, R. W., Walkup, K. R., \& Beck, B. B. (2011). Animal tool behavior: The use and manufacture of tools by animals. Baltimore: Johns Hopkins University Press.

St. Amant, R., \& Horton, T. (2008). Revisiting the definition of animal tool use. Animal Behavior, 75, 1199-1208.

Thompson, L., Campbell, R., \& Rosell, F. (2007). Tool use in a display behavior by Eurasian beavers (Castor fiber). Animal Cognition, 10, 477-482.

Van Lawick-Goodall, J. (1970). Tool-using in primates and other vertebrates. Advances in the Study of Behavior, 3, 195-249.

Van Lawick-Goodall, J. (1973). Cultural elements in a chimpanzee community. In E. Menzel (Ed.), Precultural primate behaviour, symposia of the fourth international congress of primatology, 1, 144-184.

Van Schaik, C. P., Deaner, R. O., \& Merrill, M. Y. (1999). The conditions for tool use in primates: Implications for the evolution of material culture. Journal of Human Evolution, 36, 719-741.

Whiten, A., Goodall, J., McGrew, W. C., Nishida, T., Reynolds, V., Sugiyama, Y., ...Boesch, C. (1999). Cultures in chimpanzees. Nature, 399, 682-685.

Wittiger, L., \& Sunderland-Groves, J. 1. (2007). Tool use during display behavior in wild cross river gorillas. American Journal of Primatology, 69, 1307-1311.

Xiang, Z., Liang, W., Nie, S., \& Li, M. (2013). A short note on extractive foraging behavior in gray snub-nosed monkeys. Integrative Zoology, 8, 389-394 